\title{
ZONEAMENTO BIOCLIMÁTICO DA REGIÃO SUDESTE DO BRASIL PARA O CONFORTO TÉRMICO ANIMAL E HUMANO
}

\section{LUTÉRCIA M. F. DE OLIVEIRA ${ }^{2}$, TADAYUKI YANAGI JUNIOR ${ }^{3}$, ELIZABETH FERREIRA ${ }^{4}$, LUIZ G. DE CARVALHO ${ }^{4}$, MARIANO P. DA SILVA ${ }^{5}$}

\begin{abstract}
RESUMO: O objetivo deste trabalho foi avaliar as condições de clima da região Sudeste do Brasil, por meio do índice de temperatura e umidade (ITU), e apresentar o zoneamento bioclimático relacionado ao conforto térmico animal e humano. Os valores de ITU foram estimados a partir de dados de temperatura e umidade relativa do ar para o período de 1980 a 2000. Quatro intervalos de ITU foram usados para classificar o desempenho humano (ITU < 74: conforto; $74 \leq$ ITU < 79: quente; $79 \leq$ ITU < 84: muito quente, e ITU $\geq 84$ : extremamente quente), e dois intervalos para classificar a produção animal (79 $\leq$ ITU $<84$ : perigoso e ITU $\geq 84$ : emergência). Os resultados mostraram maior risco de desconforto térmico no período de outubro a abril, comparado ao período entre maio e setembro. O período mais crítico ocorreu entre dezembro e março. Apesar de se ter observado que valores de ITU méd entre 79 e 84 ocorreram em menos de 3,6\% do tempo, humanos, bem como animais, podem sofrer algum grau de estresse térmico durante as horas mais quentes do dia, afetando ambos negativamente, produção animal e rendimento humano. Valores de ITU maiores que 84 não foram observados para a região estudada.
\end{abstract}

PALAVRAS-CHAVE: ambiente térmico, produção animal, rendimento humano.

\section{BIOCLIMATIC MAPPING OF SOUTHERN BRAZILIAN REGION FOR ANIMAL AND HUMAN THERMAL COMFORT}

\begin{abstract}
The main goal of this study was to evaluate the Southeastern Brazilian climate conditions through temperature-humidity index (THI) and to present a bioclimatic mapping relating to animal and human thermal comfort. The THI values were estimated by air-temperature and relative humidity for a period from 1980 to 2000. Four intervals of THI were used to classify human performance (THI < 74: comfort; $74 \leq \mathrm{THI}<79$ : warm; $79 \leq \mathrm{THI}<84$ : hot and THI $\geq 84$ : extremely hot) and two intervals to classify animal production $(79 \leq \mathrm{THI}<84$ : dangerous and THI $\geq 84$ : emergency). The results showed higher risk of thermal discomfort in the period from October to April, comparing to the period between May and September. The worst period was the one between December and March. Although it was observed that $\mathrm{THI}_{\text {average }}$ values among 79 and 83 occurred in less than $3.6 \%$ of the time, humans as well as animals could suffer some amount of thermal stress during the hottest period of the day, affecting negatively both animal production and human comfort. THI greater than 84 was not verified on the Southern Brazilian region.
\end{abstract}

KEYWORDS: thermal environment, animal production, human performance.

\footnotetext{
${ }^{1}$ Extraído da dissertação de mestrado do primeiro autor.

2 Mestre, Arquiteta da Prefeitura Municipal de Viçosa - MG.

${ }^{3}$ Prof. Adjunto, DEG/UFLA, Caixa Postal 3037, Lavras - MG, Fone: (0XX35) 3829.1374, yanagi@ ufla.br

${ }^{4}$ Prof. Adjunto, DEG/UFLA, Lavras - MG.

${ }^{5}$ Doutor, Pós-Doutorando no DEA/UFV, Viçosa - MG.

Recebido pelo Conselho Editorial em: 11-1-2005

Aprovado pelo Conselho Editorial em: 25-4-2006
} 


\section{INTRODUÇÃO}

O clima é um dos principais fatores que afetam a produção animal, sendo estratégico o seu conhecimento para o projeto de instalações e de sistemas de arrefecimento e para o manejo dos animais. Assim, para que os animais possam exprimir todo o seu potencial produtivo, torna-se necessário considerar a interação entre genética, nutrição, sanidade e ambiente térmico. $\mathrm{O}$ ambiente térmico, geralmente, engloba os efeitos da radiação solar, temperatura $\left(\mathrm{t}_{\mathrm{ar}}\right)$, umidade relativa (UR) do ar e velocidade do vento ( $\mathrm{v}_{\mathrm{ar}}$ ) (FALCO, 1997; BAETA \& SOUZA, 1997), sendo a combinação $\mathrm{t}_{\mathrm{ar}}$ - UR o principal condicionante para conforto térmico e o funcionamento geral dos processos fisiológicos.

Por outro lado, enquanto a ambiência animal tem recebido maior atenção por parte dos pesquisadores, poucos estudos têm sido conduzidos com relação à ambiência do trabalhador rural. Embora seja do conhecimento que altos valores de $t_{a r}$ e UR resultam em desconforto térmico, geralmente prejudicial para os seres humanos, muito pouco tem sido feito para amenizá-lo, tendo como conseqüência prejuízo à saúde dos trabalhadores rurais, tendo-se percebido que o rendimento e o risco de acidentes dos trabalhadores estão mais relacionados ao desconforto térmico.

Na tentativa de estabelecer critérios para a classificação dos ambientes, foram desenvolvidos diversos índices de conforto térmico que visam a englobar, em um único parâmetro, o efeito conjunto dos elementos meteorológicos e do ambiente construído sobre o indivíduo estudado, homem ou animal. Exemplos de índices térmicos desenvolvidos para animais são: índice de temperatura e umidade - ITU (THOM, 1959), índice de temperatura do globo negro e umidade ITGU (BUFFINGTON et al., 1981), índice de desconforto térmico - IDT (YANAGI JÚNIOR et al., 2001), índice de temperatura, umidade e velocidade do ar - ITUV (TAO \& XIN, 2003), dentre outros. Para humanos, pode-se citar o índice de estresse térmico - $\mathrm{SW}_{\text {req }}$ (BELDING \& HATCH, 1955), o índice de bulbo úmido e temperatura do globo - IBUTG (YAGLOU \& MINARD, 1957) e o próprio ITU (THOM, 1959), dentre outros.

Apesar de existirem índices de conforto térmico mais completos que o ITU, esse tem sido muito utilizado por envolver apenas informações meteorológicas normalmente disponíveis em estações meteorológicas e em bancos de dados obtidos a partir de imagens de satélite.

Assim, para o projeto e manejo de instalações para produção animal e verificação das condições de conforto térmico animal e do trabalhador rural, podem-se utilizar informações advindas de bancos de dados, principalmente meteorológicos. Esses bancos de dados podem ser trabalhados por meio da tecnologia de sistema de informações geográficas (SIG), possibilitando a geração de mapas temáticos, como, por exemplo, o zoneamento bioclimático de uma determinada região, importante para a tomada de decisão com relação à ambiência animal e humana.

Este trabalho teve o objetivo de fazer o zoneamento bioclimático da região Sudeste do Brasil, por meio do índice de temperatura e umidade (ITU).

\section{METODOLOGIA}

\section{Classificação do ambiente térmico}

Foi elaborado o mapeamento das zonas térmicas do ambiente de criação e de rendimento humano, por meio do índice de temperatura e umidade (ITU), desenvolvido por THOM (1959), visando a definir zonas de conforto térmico para pessoas, sendo posteriormente usado para avaliar o conforto térmico de animais e pessoas (eq.1).

$$
\mathrm{ITU}=\mathrm{t}_{\mathrm{bs}}+0,36 \mathrm{t}_{\mathrm{po}}+0,42
$$

em que,

$\mathrm{t}_{\mathrm{bs}}$ - temperatura de bulbo seco $\left({ }^{\circ} \mathrm{C}\right), \mathrm{e}$

$\mathrm{t}_{\mathrm{po}}$ - temperatura do ponto de orvalho $\left({ }^{\circ} \mathrm{C}\right)$, obtida pela metodologia proposta por WILHELM (1976). 
Para a definição das zonas de conforto e desconforto térmico para trabalhadores rurais, consideraram-se a taxa metabólica $\left(\mathrm{W} \mathrm{m}^{-2}\right)$ e o isolamento térmico da vestimenta, cuja resistência térmica $\left({ }^{\circ} \mathrm{C} \mathrm{W} \mathrm{m}{ }^{-2}\right.$ ) é dada em clo (clotting), em que $0,15^{\circ} \mathrm{C} \mathrm{W} \mathrm{m}^{-2}$ equivale a 1 clo. Neste estudo, considerou-se que os trabalhadores de granjas, no geral, exercem atividades moderadas, com taxa metabólica de $175 \mathrm{~W}$, trabalhando em pé, com movimentação de braços e pernas e a utilização de vestimentas leves, ou seja, com resistência térmica de 0,6 clo. Dessa forma, com base nos estudos desenvolvidos por OLGYAY (1963 e 1968), KOENIGSBERGER et al. (1977), RIVERO (1986), ESMAY \& DIXON (1986) e LAMBERTS et al. (1997) e dos parâmetros mencionados, foram definidas as seguintes zonas de conforto e desconforto térmico para trabalhadores de granjas:

ITU < 74: conforto térmico adequado;

$74 \leq$ ITU < 79: ambiente quente, no qual se inicia o desconforto térmico, podendo causar problemas de saúde e redução no rendimento do trabalhador rural;

$79 \leq$ ITU < 84: condições ambientais muito quentes, indicando perigo e podendo trazer consequiências graves à saúde do trabalhador rural, e

ITU > 84: indica condição extremamente quente, com risco muito grave à saúde do trabalhador rural.

De acordo com THOM (1959), HAHN (1982), ROSEMBERG et al. (1983), DU PREEZ et al. (1990), HUBBARD et al. (1999), CABRAL (2001) e HUHNKE et al. (2001), para aves, bovinos e suínos, os intervalos de ITU considerados são de:

79 < ITU < 84: implica condição de perigo para os animais, indicando aos produtores a necessidade de se tomarem precauções para evitar perdas na produção, e

ITU > 84: indica situação de emergência, sendo necessário que providências urgentes sejam tomadas para evitar a perda do plantel.

Para os animais, não foram feitas classificação com relação às zonas de conforto e início de desconforto, por ser variável entre as espécies. Para aves, os intervalos de ITU são idênticos aos usados para humanos; por outro lado, para bovinos de leite, valores entre 72 e 79 comprometem o desempenho produtivo e reprodutivo.

\section{Estimativa do ITU}

Para a estimativa do ITU para a região Sudeste do Brasil, foram utilizados dados do Projeto Reanálise-2, do National Centers for Environmental Prediction e do National Center for Atmospheric Research (NCEP/NCAR, 2004), compreendidos entre janeiro de 1980 e dezembro de 2001. Para este estudo, foi usada malha constituída por 90 pontos, que extrapolam a região Sudeste, propiciando maior precisão durante o processo de interpolação, sendo utilizados dez pontos igualmente espaçados para a longitude, variando de $37^{\circ} 30^{\prime} 00^{\prime \prime}$ a $54^{\circ} 22^{\prime} 30^{\prime \prime}$ oeste e nove pontos igualmente espaçados para a latitude, variando de $12^{\circ} 22^{\prime} 6^{\prime \prime}$ a $27^{\circ} 37^{\prime} 7^{\prime \prime}$ sul. Esse índice foi determinado de hora em hora para todos os dias, no período mencionado. Para tanto, a determinação dos valores horários $t_{\mathrm{ar}}$ ao longo do dia foi feita a partir dos dados de temperatura mínima do ar $\left(\mathrm{t}_{\mathrm{mín}}\right)$, temperatura do ar às $9 \mathrm{~h}$ do horário local $\left(\mathrm{t}_{9}\right)$, temperatura máxima do ar $\left(\mathrm{t}_{\mathrm{máx}}\right) \mathrm{e}$ temperatura do ar às $21 \mathrm{~h}$ do horário local $\left(\mathrm{t}_{21}\right)$, utilizando-se da metodologia proposta por ZOLNIER (1996). A escolha desse modelo para a estimativa da variação diária da temperatura baseia-se no estudo desenvolvido por LYRA et al. (2003), que mostraram o melhor ajuste obtido por esse modelo, comparado ao proposto por CAMPBELL \& NORMAN (1998). Os valores horários de UR também foram obtidos por interpolação, de acordo com a metodologia proposta por ZOLNIER (1996).

Os dados horários de $\mathrm{t}_{\mathrm{ar}}$ e UR para cada dia dos anos estudados, para cada latitude e longitude, foram usados como entrada em um programa desenvolvido em FORTRAN, para o cálculo das $\mathrm{t}_{\mathrm{ar}} \mathrm{e}$ UR horárias médias e máximas. Esses dados foram usados para a determinação da temperatura do ponto de orvalho $\left(\mathrm{t}_{\mathrm{po}}\right)$, permitindo, em seguida, o cálculo do ITU (THOM, 1959) 
para cada horário, com base na metodologia proposta por WILHELM (1976). Foram calculadas, também, as médias do número total de horas de ocorrência mensal de ITU para intervalos definidos para essa variável.

\section{Geração de mapas temáticos}

Para a geração dos mapas temáticos, utilizou-se o aplicativo SPRING - Sistema para Processamento de Informações Georreferenciadas (CÂMARA et al., 1996), e o banco de dados b_atlas_access.exe do Instituto Nacional de Pesquisas Espaciais (INPE), disponível na página www.dpi.inpe.br/spring. A interpolação dos dados, para uma grade retangular de 50 x $50 \mathrm{~km}$, foi feita utilizando-se, para os valores da cota de cada ponto, da média simples dos oito pontos mais próximos. A partir das informações georreferenciadas de ITU médio e máximo e do número de horas de ocorrência de ITU dentro dos intervalos considerados, foram geradas imagens contendo oito classes representativas dos valores de ITU e do número de horas de ocorrência para cada mês do ano. A finalização dos mapas gerados foi feita por meio do módulo SCARTA do aplicativo SPRING.

\section{RESULTADOS E DISCUSSÃO}

Após o processamento dos dados climáticos, determinação do ITU e geração dos mapas temáticos, observa-se, de janeiro a julho, a tendência de resfriamento da região, sendo posteriormente seguida por aquecimento até dezembro, mostrando a influência das estações do ano sobre o clima regional (Tabela 1 e Figuras 1 e 2).

Os valores médios de $\mathrm{ITU}_{\text {méd }}$ e ITU máx (indicado entre parênteses) para as estações do ano, verão, outono, inverno e primavera, são de 73,2 (77,4); 71,1 (75,7); 67,4 (73,0) e 70,6 (76,1), respectivamente. Assim, com base nos valores de $\mathrm{ITU}_{\text {méd }}$ e $\mathrm{ITU}_{\text {máx }}$, verifica-se que, com exceção da estação de inverno, todas as demais representam certo grau de estresse térmico, tanto para os animais quanto para os trabalhadores rurais (Tabela 1). Entretanto, quando analisados os valores máximos durante o ano, observa-se que situações de estresse térmico podem ocorrer durante todos os meses do ano.

Como se verifica pelos valores de $\mathrm{V}_{\text {máx }}$ para $\operatorname{ITU}_{\text {máx }}$ (Tabela 1), a condição de perigo para os animais pode ser observada para todos os meses do ano, exceto para o período de maio a julho, no qual o maior valor de $I_{\text {máx }}$ foi de 78,7. Durante o período de desconforto térmico para os animais devido aos valores de ITU, verifica-se a necessidade, por parte dos produtores, de tomarem precauções para evitar perdas na produção, tais como, manutenção e/ou instalação de sistemas de ventilação e/ou resfriamento evaporativo.

TABELA 1. Estatística descritiva para o índice médio de temperatura e umidade $\left(I_{T} U_{\text {méd }}\right)$ mensal e índice máximo de temperatura e umidade (ITU máx) mensal.

\begin{tabular}{|c|c|c|c|c|c|c|c|c|}
\hline \multirow{2}{*}{ Mês } & \multicolumn{4}{|c|}{$\mathrm{ITU}_{\text {méd }}$} & \multicolumn{4}{|c|}{$I_{T} U_{\text {máx }}$} \\
\hline & $\mathrm{x}$ & $\mathrm{s}$ & $\mathrm{V}_{\text {mín }}$ & $\mathrm{V}_{\text {máx }}$ & $\mathrm{X}$ & $\mathrm{S}$ & $\mathrm{V}_{\text {mín }}$ & $\mathrm{V}_{\text {máx }}$ \\
\hline janeiro & 73,4 & 1,5 & 70,7 & 74,4 & 77,5 & 1,48 & 74,6 & 81,3 \\
\hline fevereiro & 73,3 & 1,8 & 70,3 & 77,0 & 77,5 & 1,22 & 74,8 & 81,3 \\
\hline março & 72,8 & 2,0 & 68,9 & 77,0 & 76,9 & 1,37 & 74,0 & 80,9 \\
\hline abril & 71,4 & 2,4 & 65,9 & 76,0 & 76,0 & 1,65 & 72,6 & 79,3 \\
\hline maio & 69,1 & 3,2 & 61,5 & 75,0 & 74,1 & 2,28 & 68,6 & 78,7 \\
\hline junho & 67,3 & 3,4 & 59,0 & 74,0 & 72,2 & 2,91 & 66,1 & 77,9 \\
\hline julho & 66,8 & 3,2 & 58,9 & 73,0 & 72,3 & 2,84 & 65,9 & 77,7 \\
\hline agosto & 68,2 & 2,8 & 61,3 & 73,0 & 74,4 & 3,70 & 67,3 & 81,1 \\
\hline setembro & 69,3 & 3,0 & 62,0 & 75,0 & 75,2 & 4,20 & 67,1 & 82,8 \\
\hline outubro & 70,8 & 2,6 & 65,1 & 75,0 & 76,4 & 3,90 & 68,9 & 83,1 \\
\hline novembro & 71,7 & 1,9 & 67,5 & 75,0 & 76,8 & 2,90 & 70,8 & 82,3 \\
\hline dezembro & 72,8 & 1,5 & 69,7 & 76,0 & 77,1 & 2,15 & 72,8 & 82,6 \\
\hline
\end{tabular}

x - média mensal de ITU; s - desvio-padrão, $\mathrm{V}_{\text {mín }}$ - valor mínimo observado; $\mathrm{V}_{\text {máx }}$ - valor máximo observado. 
Nas Figuras 1 e 2, ilustra-se a variação do ITU $_{\text {méd }}$ e do ITU máx ao longo do ano. Os meses de maio a setembro caracterizam-se pelos menores riscos de desconforto térmico devido aos menores valores de temperatura e umidade relativa do ar, para toda a região Sudeste, indicando ser o período de menor risco à produção animal e o período no qual os trabalhadores rurais podem obter maiores rendimentos no desenvolvimento de atividades de campo, além de reduzir os riscos de acidentes causados devido ao desconforto térmico.

Conforme especificado anteriormente, não foram feitas classificações para o ambiente animal no intervalo de transição entre conforto e desconforto, por haver diferenças entre as espécies.

Os problemas relacionados aos altos valores de ITU são mais bem caracterizados por meio do ITU $_{\text {máx }}$, conforme Figura 2, em que se verificou que as mesorregiões que apresentaram problemas relacionados aos altos valores de ITU foram o oeste de São Paulo, uma parte do Alto Paranaíba e o Triângulo Mineiro, o extremo do Jequitinhonha e Vales do Mucuri e Rio Doce, bem como o norte e parte do litoral do Espírito Santo e o noroeste do Rio de Janeiro. Os resultados encontrados neste trabalho se aproximam dos obtidos por CABRAL (2001).
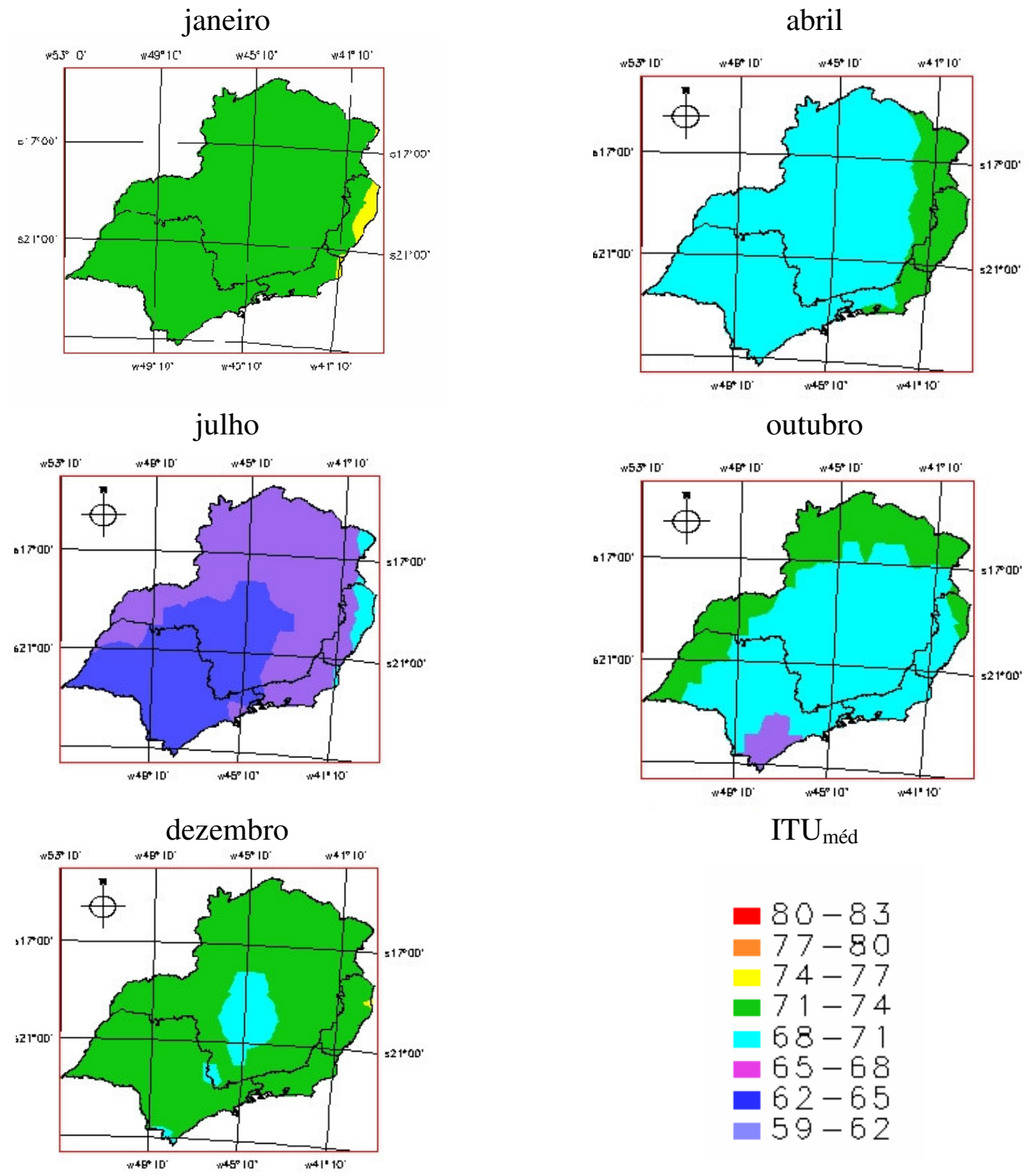

ITU méd

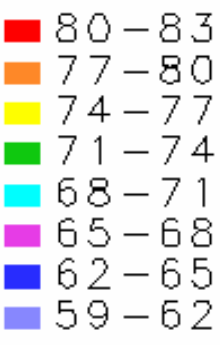

FIGURA 1. Distribuição espacial e temporal do índice médio de temperatura e umidade mensal $\left(\mathrm{ITU}_{\text {méd}}\right)$ para a região Sudeste do Brasil, no período compreendido entre os anos de 1980 e 2000. 
Com relação ao rendimento dos trabalhadores rurais ao longo do ano, verifica-se que, em parte da região Sudeste do Brasil, os trabalhadores rurais podem estar sujeitos a início de desconforto térmico $(74 \leq$ ITU $<79)$, e, em outras, o ambiente térmico é caracterizado como de condições ambientais muito quentes, o que pode trazer danos à saúde dos trabalhadores rurais (Figura 2).
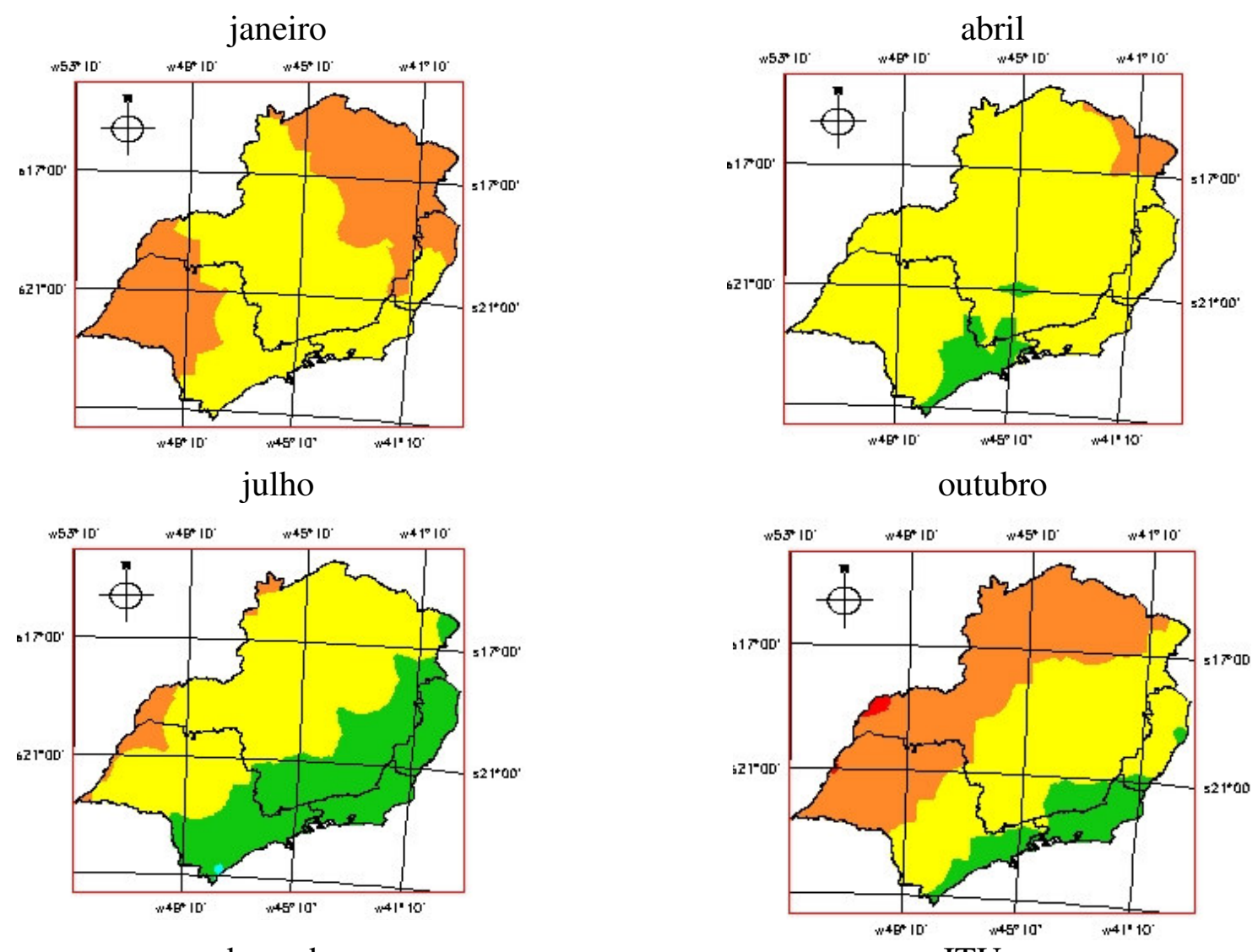

dezembro

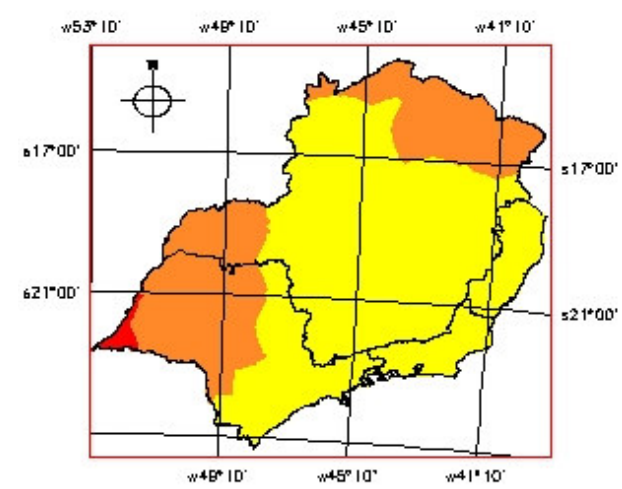

ITU $U_{\text {máx }}$

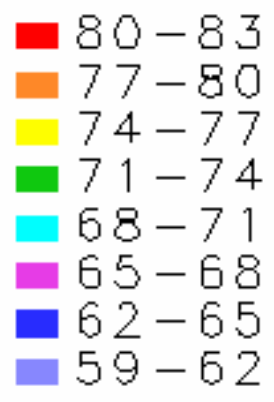

FIGURA 2. Distribuição espacial e temporal dos valores médios do índice máximo de temperatura e umidade mensal (ITU máx) na região Sudeste do Brasil, para o período compreendido entre os anos de 1980 e 2000.

Entre novembro e abril, verificou-se a ocorrência de valores de ITU que caracterizam condições de desconforto térmico em diferentes níveis, tanto para humanos quanto para animais, sendo os meses mais críticos de dezembro a março, nos quais, em pelo menos 34,6\% do total de horas do mês, ocorreram valores de ITU entre 74 e 79 (Tabela 2), que caracterizam ambiente quente para os trabalhadores rurais e inicia-se o desconforto por calor, podendo causar problemas de saúde e redução do rendimento do trabalhador. 
Entre maio e setembro, observou-se a ocorrência de ITU menor que 74 em pelo menos $80 \%$ do período e, para junho, julho e agosto, a percentagem de ocorrência foi superior a 91\% (Tabela 2). Entre maio e setembro, a ocorrência de valores de ITU entre 74 e 79 foi inferior a $15 \%$ e, entre 79 e 84 , foi inferior a 3,60\%. A distribuição desses valores de ITU nos intervalos preestabelecidos indica condição de clima ameno, sendo mais adequado para a produção animal, além de permitir aos trabalhadores de granjas rurais melhores condições de conforto, que pode ser traduzida em maiores rendimentos, reduzindo as possibilidades de riscos de acidentes devido ao desconforto gerado pelos altos valores de ITU. Situações de emergência com relação à produção animal e condições extremamente quentes para o homem, com risco à saúde do trabalhador rural, caracterizada por valores de ITU maiores que 84, não foram observadas para a região Sudeste do Brasil.

TABELA 2. Percentagem média do número de horas mensais de ocorrência de ITU médio dentro dos intervalos estudados.

\begin{tabular}{lcccc}
\hline \multirow{2}{*}{ Mês } & \multicolumn{4}{c}{ Intervalo de ITU méd } \\
\cline { 2 - 5 } & ITU $<74$ & $74 \leq$ ITU $<79$ & $79 \leq$ ITU $<84$ & ITU $\geq 84$ \\
\hline janeiro & 50,35 & $\mathbf{4 8 , 7 2}$ & 0,93 & 0,00 \\
fevereiro & 47,50 & $\mathbf{5 2 , 1 0}$ & 0,40 & 0,00 \\
março & 53,75 & $\mathbf{4 6 , 2 0}$ & 0,09 & 0,00 \\
abril & 71,43 & $\mathbf{2 8 , 5 7}$ & 0,01 & 0,00 \\
maio & 82,28 & 13,73 & 0,00 & 0,00 \\
junho & 91,47 & 4,74 & 0,00 & 0,00 \\
julho & 97,32 & 2,70 & 0,00 & 0,00 \\
agosto & 92,78 & 6,87 & 3,60 & 0,00 \\
setembro & 86,01 & 11,92 & 2,06 & 0,00 \\
outubro & 71,03 & 18,77 & 2,95 & 0,00 \\
novembro & 72,54 & $\mathbf{2 5 , 9 0}$ & 1,57 & 0,00 \\
dezembro & 61,20 & $\mathbf{3 4 , 6 0}$ & 0,97 & 0,00 \\
\hline
\end{tabular}

\section{CONCLUSÕES}

A análise dos dados de ITU com base no zoneamento bioclimático mostrou que o período entre maio e setembro se caracterizou pelos menores riscos de desconforto térmico animal e de trabalhadores rurais. Porém, podem ser observados níveis diferenciados de desconforto térmico devido às altas temperaturas e umidades relativas durante certas horas do dia para todas as mesorregiões da região Sudeste ao longo do ano, sendo que as mesorregiões com maiores problemas decorrentes do calor são o oeste de São Paulo, uma parte do Alto Paranaíba e o Triângulo Mineiro, o extremo das regiões do Jequitinhonha e Vales do Mucuri e Rio Doce (Minas Gerais), bem como o norte e parte do litoral do Espírito Santo e o noroeste e norte do Rio de Janeiro.

\section{REFERÊNCIAS}

BAÊTA, F.da C.; SOUZA, C. de F. Ambiência em edificações rurais: conforto térmico animal. Viçosa - MG: Editora UFV, 1997. 246 p.

BELDING, H.S.; HATCH, T.F. Index for evaluating heat stress in terms of resulting physiological strains. Heating, piping and air conditioning, New York, v.27, p.129-36, 1955.

BUFFINGTON, D.E. et al. Black globe-humidity index (BGHI) as comfort equation for dairy cows. Transactions of the ASAE, St. Joseph, v.24, n.3, p.711-14, 1981.

CABRAL, J.L. Mapeamento da região Sudeste do Brasil usando o ITU, para o gado de leite. 2001. 68 f. Dissertação (Mestrado em Meteorologia Agrícola) - Universidade Federal de Viçosa, Viçosa - MG, 2001. 
CÂMARA, G.; SOUZA, R.C.M.; FREITAS, U.M.; GARRIDO, J. SPRING: Integrating remote sensing and GIS by object-oriented data modelling. Computers \& Graphics, Amsterdam, v.20, n.3, p.395-403, May-Jun. 1996.

CAMPBELL, G.S.; NORMAN, J.M. An introduction to environmental biophysics. $2^{\text {nd }}$ ed. New York: Springer, 1998. $286 \mathrm{p}$.

DU PREEZ, J.D.; GIESECKE, W.H.; HATTINGH, P.J. Heat stress in dairy cattle and other livestock under Southern African conditions. I. Temperature-humidity index mean values during the four main seasons. Onderstepoort Journal Veterinary Research, Onderstepoort, v.57, n.1, p.7787, 1990.

ESMAY, M.L.; DIXON, J.E. Environmental control for agricultural buildings. West Port: AVI, 1986. 287 p.

FALCO, J. E. Bioclimatologia animal. Lavras: UFLA, 1997. 57 p.

HAHN, G.L. Compensatory performance in livestock: influences on environmental criteria. In: INTERNATIONAL LIVESTOCK ENVIRONMENT SYMPOSIUM, 2., 1982, Ames. Proceedings...p.285-294.

HUBBARD, K.G.; STOOKSBURY, D.E.; HAHN, G.L.; MADER, T.L. A climatological perspective on feedlot cattle performance and morality related to the temperature-humidity index. Journal Production Agriculture, Madison, v.12, n.4, p.650-3, 1999.

HUHNKE, R.L.; McCOWAN, L.C.; MEREZ, G.M.; HARP. S.L.; PAYTON, M.E. Determining the frequency and duration of elevated temperature-humidity index. In: ASAE ANNUAL INTERNATIONAL MEETING, 2001, Califórnia. Proceedings... St. Joseph: ASAE, 2001. 12 p. (Paper $\mathrm{n}^{\circ}$ 01-4111)

KOENIGSBERG, O.H.; INGERSOLL, T.G.; MAYHEW, A.; SZOKOLAY, S.V. Viviendas y edificios en zonas calidas y tropicales. Madri: Paraninfo, 1977. 328 p.

LAMBERTS, R.; DUTRA, L.; PEREIRA, F.O.R. Eficiência energética na arquitetura. São Paulo: PW Editores, 1997. 192 p.

LYRA, G.B.; CABRAL, S.; JÚNIOR, J.L.; SANTOS, S.N.M.; SILVA, W.C.M. Modelos empíricos para estimativa da variação diária da temperatura do ar em Piracicaba - SP. In: CONGRESSO BRASILEIRO DE AGROMETEOROLOGIA, 13., 2003, Santa Maria. Anais...p.727-8.

NATIONAL CENTERS FOR ENVIRONMENTAL PREDICTION (NCEP)/NATIONAL CENTER FOR ATMOSPHERIC RESEARCH (NCAR). Disponível em:

<http://www.cpc.ncep.nooa.gov/products>. Acesso em: 24 jul. 2004.

OLGYAY, V. Clima y arquitectura en Colombia. Cali: Carvajal, 1968. 240 p.

OLGYAY, V. Design with climate-bioclimate approach to architectural regionalism. Princeton: University Press, 1963. 190 p.

RIVERO, R. Arquitetura e clima: acondicionamento térmico natural. Porto Alegre: D.C. Luzzatto Editores, 1986. 240 p.

ROSENBERG, L. J.; BIAD, B. L.; VERNS, S. B. Human and animal biometeorology. In: Microclimate, the biological environment. New York: Wiley-Interscience Publication, 1983.

TAO, X.; XIN, H. Acute synergistic effects of air temperature, humidity, and velocity on homeostasis of market-size broilers. Transactions of the ASAE, St. Joseph, v.46, n.2, p.491-7, 2003.

THOM, E.C. The discomfort index. Weatherwise, Boston, v.12, n.1, p.57-60, 1959.

YAGLOU, C.P.; MINARD, D. Control of heat casualties at military training centers. Archives of Industrial Health, Chicago, v.16, p.302-5, 1957. 
WILHELM, L.R. Numerical calculation of psychrometric properties in SI units. Transactions of the ASAE, St. Joseph, v.19, n.2, p.318-21 e 325, 1976.

ZOLNIER, S. Avaliação de modelos para estimativa dos valores médios horários do índice de temperatura e umidade. Engenharia na Agricultura, Viçosa, v.5, n.16, p.1-17, 1996. 